\title{
Y el COVID-19 transformó al sistema educativo: reflexiones y experiencias
} por aprender

\section{And COVID-19 transformed the educational system: reflections and experiences to learn}

Julio Cabero Almenara.

Universidad de Sevilla.

cabero@us.es

Rubicelia Valencia.

Macmillan Education, México.

rubivalencia@gmail.com

\section{RESUMEN.}

La situación producida por la COVID-19 ha impactado de manera rápida y traumática el sistema educativo de todos los niveles, trasladando las clases presenciales a la modalidad virtual a distancia, y las tecnologías de la información y comunicación han tenido una gran importancia en este proceso. Este aprendizaje mediado por las tecnologías ha tenido grandes consecuencias respecto a cómo el profesor imparte la enseñanza, cómo el estudiante interactúa en dicho contexto y cómo se llevan a cabo las tareas escolares. La diferencia en el acceso de los estudiantes a las tecnologías ha tenido repercusiones y ha aumentado la brecha social. De esta situación debemos identificar una serie de variables que han dificultado el proceso: la formación del profesorado y el alumnado respecto a sus competencias digitales, la importancia asignada a diferentes tecnologías, la necesidad de poner a disposición de los alumnos tecnologías a fin de evitar la ampliación de la brecha digital, y la necesidad de transformar tanto las funciones desempeñadas por el docente como la concepción del proceso de enseñanza-aprendizaje. El aprendizaje virtual llegó para quedarse, y la atención debe ponerse ahora en el compromiso, la eficacia y la potenciación de la práctica reflexiva.

\section{PALABRAS CLAVE.}

COVID-19, formación del profesorado, educación virtual, competencia digital, brecha digital.

\section{ABSTRACT.}

The situation created by COVID-19 has had a strong and traumatic impact on the educational system of all levels, moving face-to-face classes to virtual ones at a distance. Information and communication technologies have had great significance in this process. This technologymediated learning has had relevant consequences regarding how teachers give their lessons, how students interact in that context, and how school tasks are carried out. The difference in student access to technologies has had implications, one of them being the widening of the social gap. And from this situation we must learn about a series of variables that have made the process difficult: the training of teachers and students in terms of their digital skills, the

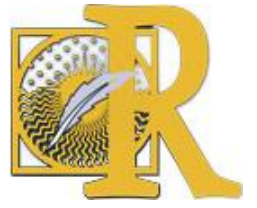

Fecha de recepción: 21-08-2020 Fecha de aceptación: 23-08-2020 
significance assigned to different technologies, the need to make technologies available to students to avoid a deepening of the digital divide, and the need to transform the functions of the teacher as well as the conception of the teaching-learning process. Virtual learning is here to stay and emphasis must now be placed on the commitment, effectiveness and empowerment of reflective practice.

\section{KEY WORDS.}

COVID-19, teacher training, virtual education, digital competence, digital divide.

\section{El "tsunami" educativo y sus profetas.}

Cuando el 31 de diciembre de 2019 sonaron las doce campanas que anunciaban el comienzo del año 2020, y gran parte del mundo pensó en todos los proyectos nacionales e internacionales en que se habían puesto muchas esperanzas y a los cuales habían denominado con dichos valores numéricos, salvo un grupo de expertos, pocas personas conocían la palabra COVID-19. Hoy, el mundo ha cambiado dramáticamente a causa de esta enfermedad, y por ella se han transformado también los sistemas educativos, que han pasado rápidamente de las situaciones presenciales a las virtuales a distancia, de la utilización de los medios impresos a la incorporación de los recursos digitales, y de la interacción directa estudiante-docente a una indirecta mediada por la tecnología.

En definitiva, ha ocurrido un verdadero "tsunami" en la educación, aunque no como el que han pretendido - en todos los tiempos - los defensores de la tesis de que la "escuela ha muerto", o como el que anunciaron esos románticos tecnológicos para quienes ciertas tecnologías - como los Massive Open Online Courses (MOOC) - cambiarían y harían irreconocibles las instituciones educativas. Este "tsunami" ha transformado las instituciones educativas en todos sus aspectos: en su estructura organizativa y en los roles desempeñados por multiplicidad de actores y herramientas (docentes y discentes, las propias tecnologías digitales, la administración, las editoriales de materiales educativos, la televisión digital, los teléfonos móviles y las redes sociales).

Lo complejo de la situación ha llevado a que las revistas científicas publiquen trabajos monográficos en los que se reflexiona sobre lo ocurrido, no solo por la complejidad observada sino por la probabilidad de que aquella se repita en un futuro cercano.

\section{Lo que el "tsunami" sacó a flote.}

El primer aspecto que puede referirse es la rapidez con la que se llevó a cabo la transformación de la acción educativa, ya que se pasó de modelos transmisores de información por parte del docente, y apoyados en una interacción directa entre docente y discente, a un modelo centrado y mediado por las tecnologías. Este hecho supuso un gran reto para todos los actores involucrados en la educación, ya que la formación a distancia rompe con tres de las variables clásicas de la educación presencial: la unidad de tiempo, de espacio y de acción, de quienes participan en la acción formativa.

Esta situación planteó diferentes problemas: si se contaba con tecnología confiable y accesible, ¿qué hacer con esa tecnología y qué metodologías aplicar, o qué contenidos transmitir, con ella?

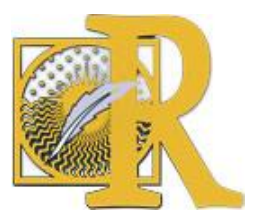

Fecha de recepción: 21-08-2020 Fecha de aceptación: 23-08-2020 
En consecuencia, lo primero que saltó a la vista fue que faltaba tecnología tanto en los centros educativos como en los hogares de los docentes y en los de los estudiantes, además de que no había conexión a internet disponible en todos los casos, ni la velocidad y estabilidad de esta eran siempre las adecuadas. La situación sería más alarmante en los países en vías de desarrollo y en las zonas rurales frente a las urbanas (Cabero y Valencia, 2019). Como señala la Unesco en un reciente documento: "En el contexto latinoamericano, las dificultades que encierra este desafío se ve incrementado por las desigualdades socioeconómicas, con las consecuentes brechas digitales, tanto de los estudiantes como del profesorado" (Rappoport, Rodríguez y Bresanello, 2020, 3).

Esta falta de tecnología ha repercutido en la profundización de la desigualdad social, en detrimento de los estudiantes con un nivel socioeconómico más desfavorecido; si bien, esta desigualdad se ha presentado incluso en los países desarrollados, ya que los estudiantes no han tenido acceso constante a las tecnologías por razones diversas, como el hecho de que sus padres estén bajando en línea y la disponibilidad de los equipos de cómputo sea limitada. A esta falta de tecnología se le podría añadir la falta de espacios adecuados que faciliten la creación de un ambiente propicio para el aprendizaje.

Es decir, la pandemia ha sacado a flote lo que diversos estudios institucionales han puesto constantemente de manifiesto: hay una "brecha digital" entre países y personas (Unesco, 2017). Y si las tecnologías digitales, especialmente internet, son clave en la sociedad del conocimiento, el no facilitar su acceso a costos razonables repercutirá en la marginación de ciertos países y colectivos humanos. Analicemos la información que ofrece el "Índice de Desarrollo Humano", elaborado por el Programa de las Naciones Unidas para el Desarrollo (PNUD); en específico, una puntuación - comprendida entre 0 y 1 - para evaluar la calidad de vida en un país, en función de las puntuaciones alcanzadas en tres variables: una vida larga y saludable, acceso a educación y un nivel de vida digno (http://hdr.undp.org/sites/default/files/hdr 2019 overview - spanish.pdf). En este informe se señala que existe una correlación alta y positiva entre un mayor índice y una mayor presencia de internet en un país.

La "brecha digital" no solo se refiere al acceso a las tecnologías, sino también al conocimiento de ellas, es decir, a qué somos capaces de hacer con las tecnologías y para qué. Si pensamos en el docente, esto se traduce en qué puede hacer él con estas herramientas, en un momento de no presencialidad, para desarrollar el proceso formativo. En gran medida, lo que se ha intentado es replicar en lo virtual lo que se ha hecho antes en lo presencial, sin reflexionar que se trata de dos contextos diferentes que requieren, tanto al docente como al estudiante, movilizar habilidades y competencias diferentes.

En favor de este procedimiento, algunos responsables de las áreas de informática de las universidades y de otros centros educativos ofrecen el argumento de que las plataformas se han utilizado bastante y han sido un éxito educativo. Esto es verdad, pero se olvidan de que la transferencia se realizaba porque los docentes subían documentos en PDF, clips de videos y materiales en PowerPoint, y los alumnos simplemente los descargaban, sin que por ello se hubieran modificado las estrategias y metodologías de enseñanza, que son diferentes en los contextos de formación virtual.

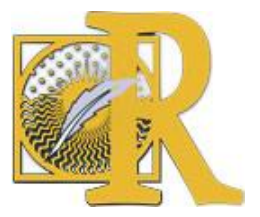

Fecha de recepción: 21-08-2020 Fecha de aceptación: 23-08-2020

Cabero, J \& Valencia, R. (2021). Y el COVID-19 transformó al sistema educativo: reflexiones y experiencias 
Respecto a los docentes, su función de "transmisores de información" ha dejado de ser significativa, en relación con otras que, en cambio, adquieren mayor importancia, a saber: consultores de información y facilitadores del aprendizaje; diseñadores de situaciones mediadas de aprendizaje; proveedores de contextos y escenografías formativas; moderadores, tutores virtuales, orientadores, y guías; evaluadores y seleccionadores de tecnologías; y curadores de contenidos (Cabero y Barroso, 2015). Estas nuevas funciones aparecen en los nuevos marcos de competencias digitales que deben poseer los docentes y que se están formulando recientemente, como es el caso del DigCompEdu de la Unión Europea (Cabero y Palacios, 2020; Cabero, Romero, Barroso y Palacios, 2020).

En contraste, lo que ha realizado el docente puede resumirse en muchas videoconferencias y en las transferencias - que hace a sus alumnos - de documentos en diferentes formatos; es decir, el docente ha seguido un modelo transmisivo sin considerar las posibilidades que la tecnología le ofrece para, por ejemplo, crear entornos colaborativos de aprendizaje y poner en acción nuevas estrategias (como el estudio de casos, la resolución de problemas, la elaboración de blogs colaborativos, la indagación y evaluación de la información existente en internet, la "caza del tesoro" o las webquests).

Esa función meramente "transmisora" implica un problema adicional, que ha sido la sobrecarga de trabajo para los estudiantes, debido a la falta de experiencia e inseguridad que la no presencialidad supone para el docente. Si bien, la sobrecarga ha alcanzado a los docentes, y ya empiezan a aparecer investigaciones que ponen de manifiesto cómo la pandemia ha incrementado su carga laboral y la jornada de trabajo misma (Allen, Rowan y Singh, 2020).

Esta complejidad ha afectado al estudiante, en primer lugar, porque en situaciones de no presencialidad, en aprendizajes mediados por la tecnología, el estudiante debe poseer altas competencias para la autorregulación de su propio proceso de aprendizaje, debe adquirir competencias para dirigirse hacia un aprendizaje autónomo y debe aprender a dialogar con el maestro a través de diferentes herramientas de comunicación sincrónicas y asincrónicas. En definitiva, debe poseer, además de la competencia anteriormente indicada, otras como la de gestión del tiempo, de la evaluación de la información, la lecto-escritora y la de colaboración. Esta última, según los hallazgos de una investigación, no figura en las preferencias de los estudiantes, aunque pueda parecer lo contrario (Cabero, 2013).

Lógicamente, otra de las competencias que debe poseer el alumno es la digital y, en este caso, muchos de los errores consisten en haber asumido, sin una fuerte reflexión, ese debate de "nativos y emigrantes digitales", según el cual se presupone que los estudiantes, por haber nacido en una época concreta, son altamente competentes para manejar las tecnologías, frente a sus docentes. La experiencia ha demostrado lo contrario: los alumnos pueden ser competentes para manejar instrumentalmente las tecnologías $-\mathrm{y}$ no todas-, pero esto no significa que sean capaces de manejarlas e incorporarlas cognitivamente a sus procesos formativos (Fundación Telefónica, 2014; Mas, 2017). Esta situación ha sido señalada por el profesorado en estos momentos de pandemia, al identificar que, aunque los estudiantes tienen un dominio instrumental de las herramientas tecnológicas y de dispositivos móviles, carecen de capacidad para la evaluación, el uso crítico y la comunicación de información (Sales, Cuevas-Cerveró y Gómez-Hernández, 2020).

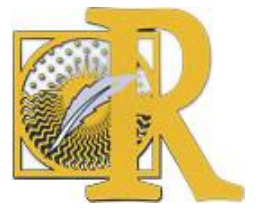

Fecha de recepción: 21-08-2020 Fecha de aceptación: 23-08-2020 Cabero, J \& Valencia, R. (2021). Y el COVID-19 transformó al sistema educativo: reflexiones y experiencias 
Como han mostrado diversas investigaciones (Castellanos, Sánchez y Calderero, 2017; Sorgo, Bartol, Dolnicar y Boh, 2017), esta situación ha llevado a que, más que "nativos digitales", se considere a los alumnos "expertos rutinarios", es decir, que saben usar las tecnologías pero no de manera inteligente, que tampoco son tan competentes en su manejo como cabría esperar y que este nivel de formación no está extendido y dominado por la única variable de la edad (Marín, Rivera, Velásquez y García, 2019; Romero-Rodríguez, ContrerasPuliod y Pérez-Rodríguez, 2019).

Respecto al terreno educativo, existen diferencias entre estudiantes y profesorado en términos del dominio de las tecnologías. Las investigaciones sobre el conocimiento que tienen los alumnos en el manejo de las TIC demuestran que no es tan cualificado como se indica desde esta teoría (Castellanos, Sánchez y Calderero, 2017; Sorgo, Bartol, Dolnicar y Boh, 2017), sobre todo en lo que se refiere específicamente a las experiencias de dominio tecnológico y formativo entre docentes y discentes. Las últimas investigaciones no apoyan la idea que presupone que los profesores ("inmigrantes digitales") son menos expertos en tecnología que los estudiantes ("nativos digitales") (Wang, Hsu, Campbell, Coster y Longhurst, 2014).

Para finalizar y, como señalan Waycott et al. (2010), a partir del estudio realizado en tres universidades australianas, solo en un pequeño porcentaje los estudiantes podrían categorizarse como "usuarios avanzados" en la utilización con gran frecuencia de una variedad de tecnologías, sin que existan grandes diferencias respecto a los docentes, quienes, según algunos estudios, muestran más competencias que los estudiantes (Flores y Del Arco, 2013; Pons, 2013). Cuando existen estas diferencias, se encuentran más en los usos de las tecnologías en el plano personal y no tanto en el educativo (Waycott et al., 2010). Otro de los mitos que han circulado últimamente respecto a las tecnologías digitales es que, por encima de cualquier otra razón, los estudiantes las prefieren para aprender. Como han demostrado Parodi, Moreno de León, Julio y Burdiles (2019) en sus investigaciones, los alumnos prefieren leer la documentación en las pantallas del ordenador cuando el contenido es liviano para su aprendizaje, pero, por el contrario, optan por trabajar con documentación impresa cuando la información es profunda y significativa para su aprendizaje y pruebas de evaluación. Como sugirió Salomon en su teoría de AME (Amount of Invested Mental Effort), las actitudes y percepciones que tienen los alumnos respecto a las tecnologías condicionan el esfuerzo mental que invierten a la hora de procesar la información: las percepciones fáciles los llevarán a invertir menos esfuerzo mental y, en consecuencia, a alcanzar menos información —como en el caso de la televisión-, mientras las percepciones de dificultad como la mostrada hacia el libro de texto- los llevarán a invertir más esfuerzo mental y a capturar de forma más profunda la información (Salomon, 1983 y 1984). Por lo tanto, no se trata sólo de la tecnología per se, sino también de la percepción que el estudiante tiene de ella.

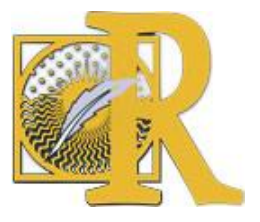

Fecha de recepción: 21-08-2020 Fecha de aceptación: 23-08-2020 
Otro problema es la falta de materiales digitales a los que tenga acceso el profesorado, que es una consecuencia de diferentes hechos: la cultura individualista en la que suele trabajar el docente -que lo lleva a producir sus propios materiales-, la fuerte dependencia que los centros han tenido de los materiales impresos, la falta de materiales digitales producidos institucionalmente y de acceso libre, y la falta de formación del profesorado.

El diseño y la producción de materiales digitales para la enseñanza exige un esfuerzo grande para el docente, y por ello es importante abordarlo de manera colaborativa: una forma de trabajo poco usual en nuestras instituciones educativas, y que se presenta como una de las competencias digitales que debe poseer el profesor del futuro (Cabero, Romero, Barroso y Palacios, 2020).

La Fundación Telefónica (2016), en una investigación que analizó centros educativos no universitarios y de diferentes niveles de enseñanza - considerados centros de "buenas prácticas" y bien preparados para enfrentarse a los retos de la educación de la sociedad del conocimiento-, encontró que todos ellos compartían una serie de principios: 1) Liderazgo y compromiso, 2) Diseño de un plan de innovación, 3) Aprendizaje basado en proyectos, 4) Aulas cooperativas, 5) Énfasis en las competencias, 6) Encaje curricular en un marco innovador, 7) Trabajo más allá del libro de texto, 8) Rol del educador como guía y activador, 9) Colaboración entre docentes y centros, 10) Redefinición de los tiempos y espacios, 11) Apertura al entorno y 12) La tecnología como vía de integración ... Y palanca de cambio.

También las relaciones del docente con la familia se han transformado, ya que los padres en esta situación han desempeñado un papel muy significativo, convirtiéndose en una extensión del docente en su domicilio. Esto ha exigido también que los padres posean ciertas competencias, no solo académicas, sino también digitales. Si bien, se han detectado algunos errores, como el intento de romper, en los domicilios, los hábitos que los hijos tenían antes de la pandemia, y pretender que éstos realicen tareas constantemente. Si en la escuela el niño o el adolescente no está ocho horas concentrado, no se le puede pedir que esté así en su domicilio.

\section{Aprendizajes por adquirir para el futuro.}

Puede ser que el COVID-19 haya acelerado muchas tendencias que iban a desarrollarse en un futuro cercano, y por ello debemos aprender de lo ocurrido.

Una de las primeras cosas que debemos aprender de esta experiencia es transformar el imaginario social y académico, que desde ciertos sectores educativos se ha creado sobre la formación virtual, presentándola como una formación de segunda categoría respecto a la presencial. Ambas formaciones tienen sus ventajas e inconvenientes, y su predilección va a venir marcada por diferentes variables que comprenden desde la edad de los estudiantes hasta la tipología de contenidos por transmitir, sin olvidarnos de las competencias que se desea alcanzar en el acto instruccional. Este imaginario, que incluso en la actualidad es defendido por determinadas autoridades académicas — con una defensa acérrima de la vuelta, lo antes posible, a la presencialidad, incluso en contextos universitarios-, va a ir calando en los docentes y, como sabemos, las creencias que se tengan sobre la enseñanza condicionarán el comportamiento que tendremos en ella. De forma que, si lamentablemente,

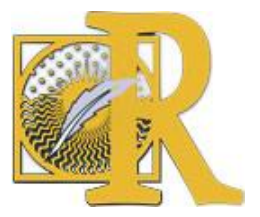

Fecha de recepción: 21-08-2020 Fecha de aceptación: 23-08-2020 
la situación se agrava, y hay que regresar al confinamiento, un grupo de profesores habrá asumido ya la idea de que lo que van a realizar es una acción educativa de segunda categoría.

Lo importante no es que la formación sea presencial o a distancia, sino que sea de calidad. Una cuestión es la distancia "física" y otra la "cognitiva", y lo que repercute en la calidad de la formación es que la segunda sea mínima. Esto puede garantizarse con las diferentes herramientas de comunicación, sincrónicas y asincrónicas, que puede movilizar el docente en la formación virtual. Lo importante, como sugirieron en su momento Garrison y Anderson (2010), es que el docente garantice tres tipos de presencia en la formación virtual: cognitiva, socialy docente. En la tabla 1, se presenta el significado que, para los autores citados, tienen estos tres tipos de presencia.

Tabla 1. Definiciones de los tipos de presencia que debe desarrollar el docente en acciones formativas (Garrison y Anderson, 2010) (Fuente: elaboración propia).

\begin{tabular}{|l|l|}
\hline Tipo de presencia & \multicolumn{1}{|c|}{ Conceptualización } \\
\hline Cognitiva & $\begin{array}{l}\text { "se refiere a los resultados educativos pretendidos y conseguidos. } \\
\text { Entendemos la presencia cognitiva «como el punto hasta el cual los } \\
\text { estudiantes son capaces de construir significado mediante la reflexión } \\
\text { continua en una comunidad de investigación." (Garrison y Anderson, 2010, } \\
50) . \text { Es, por tanto, un proceso de pensamiento crítico. Y está estrechamente } \\
\text { relacionado con el contexto donde el aprendizaje se produce. Implican la } \\
\text { utilización de habilidades cognitivas de orden superior, que se mejoran } \\
\text { cuando se llevan a cabo acciones de aprendizaje cooperativo. }\end{array}$ \\
\hline Social & $\begin{array}{l}\text { "la capacidad de los participantes en una comunidad de investigación de } \\
\text { proyectarse a sí mismos social y emocionalmente, como personas «reales" } \\
\text { (es decir, su personalidad plena), mediante los medios de comunicación en } \\
\text { uso" (Garrison y Anderson, 2010, 50). Un alto uso de la presencia social } \\
\text { repercute positivamente en el aumento de la presencia cognitiva. }\end{array}$ \\
\hline Docente & $\begin{array}{l}\text { "La presencia docente es definida como la acción de diseñar, facilitar y } \\
\text { orientar los procesos cognitivo y social con el objetivo de obtener resultados } \\
\text { educativos personalmente significativos y de valor docente... La presencia } \\
\text { docente es importante para estructurar las actividades educativas, controlar } \\
\text { los debates y equilibrar la calidad y cantidad de las aportaciones de los } \\
\text { participantes. Pero el profesor, además, debe animar la reflexión y el discurso } \\
\text { mediante la crítica constructiva" (Garrison y Anderson, 2010, 51-52). }\end{array}$ \\
\hline
\end{tabular}

Asociado a esta idea encontramos también el miedo de los propios docentes a desaparecer, un sentimiento que ha surgido cada vez que ha aparecido una tecnología que se consideraba que podría ser como el bálsamo de Fierabrás de Don Quijote, que curaba todos los males y que, en nuestro caso, sería la panacea que resolvería todos los problemas de fracaso escolar.

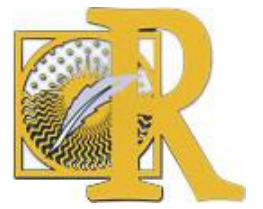

Fecha de recepción: 21-08-2020 Fecha de aceptación: 23-08-2020

Cabero, J \& Valencia, R. (2021). Y el COVID-19 transformó al sistema educativo: reflexiones y experiencias 
Pasó con las máquinas de enseñar, continuó con la televisión y ahora lo asociamos a internet. El docente nunca desaparecerá; es él quien, con su estrategia de utilización, le da sentido y vida pedagógica a la tecnología. Lo que sí pasará es que deberá cambiar roles y funciones, como ya ha hecho en otros momentos históricos (por ejemplo, cuando apareció el libro de texto).

La importancia que ha tenido la tecnología - y su falta en algunos casos-en esta situación, puede llevarnos a caer en el error de creer que todo lo resolveremos con mayor presencia tecnológica: el solucionismo tecnológico al que se refiere Morozov (2015). La tecnología es necesaria, y más si queremos disminuir las desigualdades educativas, ya que no todos los estudiantes han tenido recursos en sus casas ni han tenido conexión a internet estable y de calidad. Pero también deberemos abordar otros aspectos, como son la redefinición del papel del docente en estos nuevos contextos formativos mediados por las tecnologías, el acceso a contenidos de calidad y la adaptación de las estructuras organizativas de las instituciones escolares a estas nuevas situaciones.

Sobre los contenidos, cada vez hay más sitios institucionales donde los docentes pueden tener acceso a estos recursos de calidad. En un reciente documento elaborado por Rappoport, Rodríguez y Bresanello (2020) para la Unesco -y al cual remitimos al lector interesado-, se presenta una gran diversidad de esos sitios creados por diferentes Ministerios de Educación de países Latinoamericanos. Además, a nivel universitario contamos con diferentes experiencias como la del "Secretariado de Recursos Audiovisuales y Nuevas Tecnologías" de la Universidad de Sevilla (http://sav.us.es).

Otro aspecto que no debe olvidarse es el de la formación del profesorado en tecnologías digitales, ya que la situación ha demostrado que los docentes, de todos los niveles educativos, no siempre son lo suficientemente habilidosos en competencias digitales para la creación de entornos formativos enriquecidos por las tecnologías, y en este aspecto urge establecer planes formativos que se aborden desde modelos diferentes a los usualmente utilizados y desde la perspectiva de nuevos marcos competenciales (Cabero y Martínez, 2019; Cabero, Romero, Barroso y Palacios, 2020).

El 22 de mayo de 2018, el Consejo de la Unión Europea (2018) estableció una recomendación relativa a las competencias clave para el aprendizaje permanente, señalando como una de ellas a la digital, que entraña el uso seguro y crítico de las tecnologías de la sociedad de la información para el trabajo, el ocio y la comunicación (https://eur-lex.europa.eu/legalcontent/ES/TXT/PDF/?uri=CELEX:32018H0604(01)\&from=EN). Con esto, lo que se quiere es hacer una llamada de atención a los estudiantes y padres de familia para que alcancen ciertos niveles de competencia digital que les permitan desenvolverse en los nuevos escenarios tecnológicos. No pensemos, por lo tanto, que son únicamente los docentes quienes deben poseer tal capacitación.

Otro aspecto importante es que docentes y familias mantengan una constante comunicación entre sí. Si desgraciadamente regresamos a una situación de confinamiento, o de doble presencialidad, el papel de los padres será clave para facilitar el proceso de instrucción de los estudiantes y, desde esta perspectiva, las acciones de asesoramiento que realicen los docentes serán claves. Como señalan Fernández y Vázquez (2016, 153), "Más allá de una metáfora ingeniosa sobre las actitudes generacionales, la distinción alumnos-nativos /



Fecha de recepción: 21-08-2020 Fecha de aceptación: 23-08-2020 
docentes-inmigrantes es un error y entraña el riesgo de la inacción. Los alumnos necesitan que la escuela fomente y oriente su alfabetización digital; los profesores tienen la obligación profesional de formarse para hacerlo".

\section{A manera de conclusión.}

Para finalizar este trabajo queremos aportar una serie de conclusiones. En primer lugar, que es mejor dialogar con las tecnologías que tener una posición de rechazo frente a ellas, en especial cuando se han presentado como opción que permite efectuar acciones educativas de calidad. Por otra parte, que la "situación producida por la COVID-19 nos ha ofrecido una oportunidad para comprender que otra modalidad de formación es posible, pero que para ello deberemos reflexionar sobre diferentes aspectos que van desde la formación en competencias digitales de profesores y estudiantes, la transformación de los roles docentes y el garantizar la equidad social de acceso a las tecnologías" (Cabero, 2020, 3). Asimismo, que el aprendizaje virtual llegó para quedarse y que la atención debe ponerse ahora en el compromiso, la eficacia y la potenciación de la práctica reflexiva. $Y$ finalmente, que es necesario abordar la problemática de la formación en competencias digitales, tanto por parte del profesorado como del alumnado.

\section{Referencias.}

- Allen, J., Rowan, L. y Singh, P. (2020). Teaching and teacher education in the time of COVID-19. Asia-Pacific Journal of Teacher Education, 48(3), 233-236. https://doi.org/10.1080/1359866X.2020.1752051

- Cabero, J. (2020). Aprendiendo del tiempo de la COVID-19. Revista Electrónica Educare (Educare Electronic Journal), 24(Suplemento Especial), 1-3.

- Cabero, J. (dir) (2013). Redes sociales para la formación ¿Cómo las perciben los alumnos universitarios en sus aulas? Sevilla: Grupo de Investigación Didáctica. Recuperado de: https://grupotecnologiaeducativa.es/images/LIBROS/redes2014.pdf

- Cabero, J. y Barroso, J. (coords.) (2015). Nuevos retos en tecnología educativa. Madrid: Síntesis.

- Cabero, J. y Martínez, A. (2019). Las tecnologías de la Información y Comunicación y la formación inicial de los docentes. Modelos y competencias digitales. Profesorado. Revista de Currículum y Formación del profesorado, 23(3), 47-268. https://doi.org/10.30827/profesorado.v23i3.9421

- Cabero, J. y Palacios, A. (2020). Marco europeo de competencia digital docente "DigCompEdu" y cuestionario "DigCompEdu Check-in". Edmetic. Revista de Educación Mediática y TIC, 9(1), 213-23.

- Cabero, J. y Valencia, R. (2019). TIC para la inclusión: una mirada desde Latinoamérica. Aula Abierta, 48(2), 139-146. DOI: https://doi.org/10.17811/rifie.48.2.2019.139-146.

- Cabero, J., Romero, R., Barroso, L. y Palacios, A. (2020). Marcos de competencias digitales docentes y su adecuación al profesorado universitario y no universitario. Revista Caribeña de Investigación Educativa, (RECIE), 4(2), 137-158.

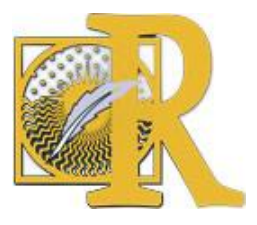

Fecha de recepción: 21-08-2020 Fecha de aceptación: 23-08-2020 
- Castellanos, A., Sánchez, C. y Calderero, J. (2017). Nuevos modelos tecnopedagógicos. Competencia digital de los alumnos universitarios. Revista Electrónica de investigación Educativa, 19(1), 1-9.

- Corbera, E., Anguelovski, I., Honey-Rosés, J. y Ruiz-Mallén, I. (2020). Academia in the time of COVID-19: Towards an ethics of care. Planning Theory \& Practice, 21(2), 191-199. https://doi.org/10.1080/14649357.2020.1757891

- Fernández, M. y Vázquez, S. (2016). La larga y compleja marcha del CLIP al CLIP. Escuela y profesorado en el nuevo entorno digital. Madrid: Fundación Telefónica-Ariel.

- Flores, O. y Del Arco, I. (2013). Nativos digitales, inmigrantes digitales: rompiendo mitos. Un estudio sobre el dominio de las TIC y estudiantado de la Universidad de Lleida. Bordón, 65 (2), 59-74.

- Fundación Telefónica (2014). 20 Claves Educativas para 2020- ¿Cómo debería de ser la educación del siglo XXI? Madrid: Fundación Telefónica.

- Fundación Telefónica (2016). Prepara tu escuela para la sociedad digital. Madrid: Fundación Telefónica.

- Garrison, D.R. y Anderson, T. (2005). El e-learning en el siglo XXI. Barcelona: Octaedro.

- Marín, I., Rivera, D., Velásquez, A. y García, R. (2019). Competencias mediáticas en Marín, V. (2013). La competencia digital de los estudiantes: elemento clave para el desenvolvimiento en la sociedad de la información. En J. Barroso \& J. Cabero (Coords.). Nuevos escenarios digitales (pp. 37-55). Madrid: Pirámide.

- Mas, X. (2017). El tejido de Weiser. Claves, evolución y tendencias de la educación digital. Barcelona: OuterEDU.

- Morozov (2015). La locura del solucionismo tecnológico. Buenos Aires: Katz Ediciones.

- Parodi, G., Moreno de León, T., Julio, C. y Burdiles, G. (2019). Generación Google o Generación Gutenberg: Hábitos y propósitos de lectura en estudiantes universitarios chilenos. Comunicar, 58, 85-94. DOI https://doi.org/10.3916/C58-2019-08.

- Pons, B. (2013). Disseny d'un programa de capacitació en competëncies bäsiques TIC per alumnes de secundaria. Palma de Mallorca, Universitat de les Illes Balears, tesis doctoral inédita.

- Rappoport, S., Rodríguez, M.S., y Bresanello, M. (2020). Enseñar en tiempos de COVID19 Una guía teórico-práctica para docentes. Montevideo: Unesco.

- Romero-Rodríguez, P., Contreras-Pulido, L. y Pérez-Rodríguez, M. (2019). Media competencies of university professors and students. Comparison of levels in Spain, Portugal, Brazil and Venezuela / Las competencias mediáticas de profesores y estudiantes universitarios. Comparación de niveles en España, Portugal, Brasil y Venezuela. Cultura y Educación, 31(2), 326-368, DOI: 10.1080/11356405.2019.1597564.

- Sales, D., Cuevas-Cerveró, A., y Gómez-Hernández, J.A. (2020). Perspectives on the information and digital competence of Social Sciences students and faculty before and during lockdown due to COVID-19. Profesional de la información, 29(4), e290423. https://doi.org/10.3145/epi.2020.jul.23

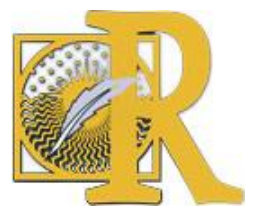

Fecha de recepción: 21-08-2020 Fecha de aceptación: 23-08-2020 
- Salomon, G. (1983). The Differential Investment of Mental Effort in Learning from Different Sources. Educational Psychologist, 18(1), 42-50.

- Salomon, G. (1984). Television is "easy" and print is "tough": The differential investment of mental effort in learning as a function of perceptions and attributions. Journal of Educational Psychology, 76(4), 647-658.

- Sorgo, A., Bartol, Th., Dolnicar, D. y Boh, B. (2017). Attributes of digital natives as predictors of information literacy in higher education. British Journal of Educational Technology, 48(3), 749-767.

- Unesco (2017). Sociedad digital: brechas y retos para la inclusión digital en América Latina y el Caribe. Montevideo: Unesco.

- Wang, Sh., Hsu, H., Campbell, T., Coster D. y Longhurst, M. (2014). An investigation of middle school science teachers and students' use of technology inside and outside of classrooms: considering whether digital natives are more technology savvy than their teachers. Education Tech Research Dev, 62, 637-662. DOI 10.1007/s11423-014-9355-4.

- Waycott, J., Bennett, S., Kennedy, G., Dalgano, B. y Gray, K. (2010). Digital divides? Student and staff perceptions of information and communication technologies. Computers \& Education, 54, 1202-1211. 